\title{
Effective collision strengths for electron impact excitation of $\mathrm{C}_{\|}{ }^{\star}$
}

\author{
N. J. Wilson, K. L. Bell, and C. E. Hudson \\ Department of Applied Mathematics and Theoretical Physics, The Queen's University of Belfast, Belfast BT7 1NN, \\ Northern Ireland \\ e-mail: c.hudson@am.qub.ac.uk
}

Received 18 August 2004 / Accepted 8 November 2004

\begin{abstract}
We extend a previous calculation of effective collision strengths for electron impact excitation of the boron-like ion C II. The earlier work was restricted to the excitation within the $2 s^{2} 2 p$ ground configuration and the sixteen $2 s^{2} 2 p-2 s 2 p^{2}$ fine-structure transitions whilst the present paper considers all fine-structure transitions among the levels arising from the lowest $16 L S$ states. The collisional cross sections are computed in the multichannel close-coupling $R$-matrix approximation, in which sophisticated configuration-interaction wave functions are used to represent the target states. The 16 states included have the following basis configurations: the $2 s^{2} 2 p$ ground state, four $2 \mathrm{~s} 2 \mathrm{p}^{2}$ states, three $2 \mathrm{p}^{3}$ states, one $2 \mathrm{~s} 2 \mathrm{p} 3 \mathrm{~s}$ state as well as the $2 \mathrm{~s}^{2} 3 l(l=\mathrm{s}, \mathrm{p}, \mathrm{d})$ and $2 \mathrm{~s}^{2} 4 l(l=\mathrm{s}, \mathrm{p}, \mathrm{d}, \mathrm{f})$ states. The $16 L S$ target states correspond to 30 fine-structure levels and lead to a total of 435 fine-structure transitions. In this paper we tabulate effective collision strengths, obtained by averaging the electron collision strengths over a Maxwellian distribution of velocities, for electron temperatures in the range given by $\log _{10} T_{\mathrm{e}}(\mathrm{K})=$ 3.0-5.5, suitable for most astrophysical applications and laboratory plasma diagnostics. Significant differences from a previous calculation are noted and comparisons are made with some recent experimental data.
\end{abstract}

Key words. atomic processes - line: formation - methods: analytical

\section{Introduction}

It is well established that carbon is an element of major astrophysical significance. In particular, emission lines of the boron-like C II ion occur frequently in astrophysical objects. For example, lines have been observed in $N$-type carbon stars (Johnson et al. 1995), in OB stars (Trapero et al. 1996), planetary nebulae (Hyung et al. 2001) and in molecular cloud/H II complexes (Boreiko \& Betz 1995). Collision strength data are used to infer properties of the plasma, such as electron temperature and/or density (Czyzak et al. 1986). It is for this reason that accurate collision strength data are needed for use by modellers.

Recently, a 16 -state $R$-matrix calculation has been performed for C II by two of the present authors (Wilson \& Bell 2002). In this work, the excitation within the $2 s^{2} 2 p$ ground configuration and the sixteen $2 \mathrm{~s}^{2} 2 \mathrm{p}-2 \mathrm{~s} 2 \mathrm{p}^{2}$ fine-structure transitions were considered. However emission lines due to other excitations, such as the $2 \mathrm{~s} 2 \mathrm{p}^{2}{ }^{2} \mathrm{~S}-2 \mathrm{~s}^{2} 3 \mathrm{p}{ }^{2} \mathrm{P}^{\mathrm{o}}$ transition occur in planetary nebulae (Vassiliadis et al. 1998) and in laboratory plasmas (Arad et al. 2000). Since these lines were not considered by Wilson \& Bell (2002), and given the likelihood that

* Table 2 is only available in electronic form at the CDS via anonymous ftp to cdsarc.u-strasbg.fr $(130.79 .128 .5)$ or via http://cdsweb.u-strasbg.fr/cgi-bin/qcat?J/A+A/432/731 collision strength data are required for other transitions, it is clearly desirable that a comprehensive tabulation of accurate collision strength data is available for use in application.

A brief review of earlier theoretical calculations, generally focusing on a limited number of transitions was given in Wilson $\&$ Bell (2002) and will not be repeated here. However, it is useful to compare with previous work, and for this purpose we will consider the effective collision strengths of Blum \& Pradhan (1992), who performed a 10-state $R$-matrix calculation, resulting in data at a number of temperatures for 153 fine-structure transitions. We also compare with the crossed beams experimental results of Williams et al. (1998) and the merged beams results of Smith et al. (1996) for the $2 \mathrm{~s}^{2} 2 \mathrm{p}^{2} \mathrm{P}^{\mathrm{o}}-2 \mathrm{~s} 2 \mathrm{p}^{2} \mathrm{D}$ excitation.

Improved computing capabilities now enable larger calculations to be undertaken and so the aim of this paper is to present Maxwellian-averaged collision strengths for the $435 \mathrm{~J}$-resolved fine-structure transitions arising from the inclusion of $16 L S$ target states in the calculation at a number of temperatures of interest to astrophysicists and plasma physicists. The increased number of target states leads to additional channel-coupling effects manifesting themselves in the cross sections obtained as resonances. Care has been taken in the present work to fully delineate these resonances, since they can lead to a considerable enhancement of the collision rates. As a 
Table 1. $L S$ energies relative to the $2 \mathrm{~s}^{2} 2 \mathrm{p}^{2} \mathrm{P}^{\mathrm{o}}$ ground state of C II. Also noted is how the $L S$ states split into $J$-levels and assignments of level indices to the target states are given. The observed values are the experimental energy levels compiled by NIST.

\begin{tabular}{|c|c|c|c|c|c|}
\hline$\overline{L S}$ state & $\begin{array}{l}\text { Present } L S \\
\text { energy(Ry) }\end{array}$ & $\begin{array}{c}\text { Observed } L S \\
\text { energy(Ry) }\end{array}$ & $\begin{array}{l}\text { No. split } \\
\text { levels }\end{array}$ & $\bar{J}$ level & $\begin{array}{l}\text { Level } \\
\text { index }\end{array}$ \\
\hline \multirow[t]{2}{*}{$2 \mathrm{~s}^{2} 2 \mathrm{p}^{2} \mathrm{P}^{\mathrm{o}}$} & 0.00000 & 0.00000 & 2 & $1 / 2$ & 1 \\
\hline & & & & $3 / 2$ & 2 \\
\hline \multirow[t]{3}{*}{$2 \mathrm{~s} 2 \mathrm{p}^{2}{ }^{4} \mathrm{P}$} & 0.38727 & 0.39179 & 3 & $1 / 2$ & 3 \\
\hline & & & & $3 / 2$ & 4 \\
\hline & & & & $5 / 2$ & 5 \\
\hline \multirow[t]{2}{*}{$2 \mathrm{~s} 2 \mathrm{p}^{2}{ }^{2} \mathrm{D}$} & 0.68854 & 0.68282 & 2 & $3 / 2$ & 6 \\
\hline & & & & $5 / 2$ & 7 \\
\hline $2 \mathrm{~s} 2 \mathrm{p}^{2}{ }^{2} \mathrm{~S}$ & 0.88923 & 0.87893 & 1 & $1 / 2$ & 8 \\
\hline \multirow[t]{2}{*}{$2 \mathrm{~s} 2 \mathrm{p}^{2}{ }^{2} \mathrm{P}$} & 1.02281 & 1.00795 & 2 & $1 / 2$ & 9 \\
\hline & & & & $3 / 2$ & 10 \\
\hline $2 s^{2} 3 s^{2} S$ & 1.06269 & 1.06158 & 1 & $1 / 2$ & 11 \\
\hline \multirow[t]{2}{*}{$2 \mathrm{~s}^{2} 3 \mathrm{p}^{2} \mathrm{P}^{\mathrm{o}}$} & 1.19905 & 1.20004 & 2 & $1 / 2$ & 12 \\
\hline & & & & $3 / 2$ & 13 \\
\hline $2 p^{3}{ }^{4} S^{o}$ & 1.29715 & 1.29386 & 1 & $3 / 2$ & 14 \\
\hline \multirow[t]{2}{*}{$2 s^{2} 3 d^{2} D$} & 1.32693 & 1.32597 & 2 & $3 / 2$ & 15 \\
\hline & & & & $5 / 2$ & 16 \\
\hline \multirow[t]{2}{*}{$2 \mathrm{p}^{3}{ }^{2} \mathrm{D}^{\mathrm{o}}$} & 1.38334 & 1.37074 & 2 & $3 / 2$ & 17 \\
\hline & & & & $5 / 2$ & 18 \\
\hline $2 s^{2} 4 s^{2} S$ & 1.43097 & 1.43244 & 1 & $1 / 2$ & 19 \\
\hline \multirow[t]{2}{*}{$2 s^{2} 4 p^{2} P^{o}$} & 1.48005 & 1.48063 & 2 & $1 / 2$ & 20 \\
\hline & & & & $3 / 2$ & 21 \\
\hline \multirow[t]{3}{*}{$2 \mathrm{~s} 2 \mathrm{p}\left({ }^{3} \mathrm{P}^{\mathrm{o}}\right) 3 \mathrm{~s}{ }^{4} \mathrm{P}^{\mathrm{o}}$} & 1.52144 & 1.52152 & 3 & $1 / 2$ & 22 \\
\hline & & & & $3 / 2$ & 23 \\
\hline & & & & $5 / 2$ & 24 \\
\hline \multirow[t]{2}{*}{$2 s^{2} 4 d^{2} D$} & 1.52981 & 1.53167 & 2 & $3 / 2$ & 25 \\
\hline & & & & $5 / 2$ & 26 \\
\hline \multirow[t]{2}{*}{$2 \mathrm{p}^{3}{ }^{2} \mathrm{P}^{\mathrm{o}}$} & 1.54761 & 1.53731 & 2 & $1 / 2$ & 27 \\
\hline & & & & $3 / 2$ & 28 \\
\hline \multirow[t]{2}{*}{$2 \mathrm{~s}^{2} 4 \mathrm{f}^{2} \mathrm{~F}^{\mathrm{o}}$} & 1.53640 & 1.53946 & 2 & $5 / 2$ & 29 \\
\hline & & & & $7 / 2$ & 30 \\
\hline
\end{tabular}

consequence, a large amount of accurate new collisional atomic data becomes available for the $\mathrm{C}$ II ion. The range of temperatures considered is appropriate for many astrophysical applications, including the study of gaseous nebulae as well as solar and laboratory type plasmas.

\section{The calculation}

We employ the multi-channel $R$-matrix method as described by Berrington et al. (1987) to compute the collision strengths $\Omega_{\text {if }}$ for electron impact excitation of C II. The relationship between $\Omega_{\text {if }}$ and the excitation cross section $\sigma_{\text {if }}$ for an initial state $\mathrm{i}$ and a final state $\mathrm{f}$ can be expressed by the equation

$\sigma_{\text {if }}=\Omega_{\text {if }} \frac{\pi a_{0}^{2}}{\omega_{\mathrm{i}} k_{\mathrm{i}}^{2}}$

in which $a_{0}$ is the Bohr radius, $\omega_{\mathrm{i}}$ is the statistical weight of the initial target level and $k_{\mathrm{i}}^{2}$ is the incident kinetic energy in rydberg. The 16 lowest $\mathrm{C}$ II states, namely $2 \mathrm{~s}^{2} 2 \mathrm{p}^{2} \mathrm{P}^{\mathrm{o}} ; 2 \mathrm{~s} 2 \mathrm{p}^{2}{ }^{4} \mathrm{P}$, ${ }^{2} \mathrm{D},{ }^{2} \mathrm{~S},{ }^{2} \mathrm{P} ; 2 \mathrm{~s}^{2} 3 \mathrm{~s}^{2} \mathrm{~S} ; 2 \mathrm{~s}^{2} 3 \mathrm{p}^{2} \mathrm{P}^{\mathrm{o}} ; 2 \mathrm{~s}^{2} 3 \mathrm{~d}^{2} \mathrm{D} ; 2 \mathrm{p}^{34} \mathrm{~S}^{\mathrm{o}},{ }^{2} \mathrm{D}^{\mathrm{o}},{ }^{2} \mathrm{P}^{\mathrm{o}}$; $2 \mathrm{~s} 2 \mathrm{p}\left({ }^{3} \mathrm{P}^{\mathrm{o}}\right) 3 \mathrm{~s}^{4} \mathrm{P}^{\mathrm{o}} ; 2 \mathrm{~s}^{2} 4 \mathrm{~s}^{2} \mathrm{~S} ; 2 \mathrm{~s}^{2} 4 \mathrm{p}^{2} \mathrm{P}^{\mathrm{o}} ; 2 \mathrm{~s}^{2} 4 \mathrm{~d}^{2} \mathrm{D} ; 2 \mathrm{~s}^{2} 4 \mathrm{f}^{2} \mathrm{~F}^{\mathrm{o}}$, are included in the calculation. Each target state is represented by a configuration-interaction wavefunction expansion, constructed from the following set of orthogonal one-electron basis functions: $1 \mathrm{~s}, 2 \mathrm{~s}, 2 \mathrm{p}, 3 \mathrm{~s}, 3 \mathrm{p}, 3 \mathrm{~d}, 4 \mathrm{~s}, 4 \mathrm{p}, 4 \mathrm{~d}, 4 \mathrm{f}, \overline{5 \mathrm{~s}}, \overline{5 \mathrm{p}}, \overline{5 \mathrm{~d}}, \overline{5 \mathrm{f}}$. The radial part of each orbital is expressed as

$P_{n l}(r)=\sum_{j n l} c_{j n l} \frac{\left[2 \zeta_{j n l}\right]^{I_{j n l}+1 / 2}}{\left[\left(2 I_{j n l}\right) !\right]^{1 / 2}} r^{I_{j n l}} \exp \left(-\zeta_{j n l} r\right)$.

The parameters used to describe these orbitals are available in Wilson \& Bell (2002) and will not be reproduced here. The wavefunctions for the C II target states are constructed using configurations generated from the replacement of two electrons in the basis distributions $2 s^{2} 2 p$ and $2 s 2 p^{2}$, the $1 \mathrm{~s}$ shell being kept closed. The non-Hartree-Fock orbitals and the target-state wavefunctions are obtained using the CIV3 program of Hibbert (1975).

A useful test of the accuracy of the wavefunctions employed is a comparison of computed energy levels with available experimental data. In Table 1, we compare the present target state energies (in rydbergs) for C II relative to the $2 s^{2} 2 p^{2} \mathrm{P}^{\mathrm{o}}$ ground state with the critically evaluated data from the NIST database at http: //www . physics . nist.gov averaged in accordance with their statistical weights. The agreement between the present theory and observed values is excellent and we thus conclude that the wavefunctions employed are 
of sufficient accuracy for the $R$-matrix collision strength calculation. In Table 1 we also present the number of $J$ levels into which each of the $16 L S$ target states is split together with the 30 values of $J$ and the assignment to each $J$ level of an in$\operatorname{dex}(1$ to 30$)$.

Using these target state wavefunctions, the procedure outlined by Burke \& Robb (1975) is used for the expansion of the ion-plus-electron (or $(N+1)$-electron) system. The $R$-matrix radius is taken to be 28.8 atomic units and a zero logarithmic derivative is imposed at this boundary. For each orbital angular momentum, 40 Schmidt-orthogonalized continuum orbitals were included, ensuring that a converged collision strength was obtained for the energy range considered. The correct positioning of resonances relative to the target states included in the calculation was ensured by adjusting our theoretical energies to the NIST values, prior to diagonalization of the Hamiltonian matrix. The energy levels for the two highest $L S$ states included in this calculation were adjusted to their arithmetic mean, $1.53839 \mathrm{Ry}$, since the energy order of these close-lying states differs from that in the NIST database.

We included all partial waves with $L \leq 12$ for both even and odd parities and doublet, quartet and sextet multiplicities. This ensured that the collision strengths for the optically forbidden transitions had converged. For dipole-allowed transitions, however, it was necessary to consider the effect of higher partial waves with $L>12$ because they have a significant effect upon the collision strengths. We assume the partial collision strengths form a geometric series expansion, with a geometric scaling factor given by the ratio of two adjacent terms. This "topping-up" procedure has been used successfully in similar calculations of Bell \& Ramsbottom (2000), and Ramsbottom et al. (2001).

For small energy intervals between fine-structure levels, it is possible to recouple the matrices obtained from nonrelativistic calculations in $L S$ coupling to obtain collision strengths between these levels. Above all the thresholds the effects of intermediate coupling in the target can be included by using this method. The present work utilizes the program of Saraph (1978) to undertake this recoupling procedure. Further details of the coupling scheme are provided by Bell \& Ramsbottom (2000).

Effective collision strengths $\Upsilon_{\text {if }}$ for a particular electron temperature $T_{\mathrm{e}}$ (in Kelvin) were obtained by averaging the collision strengths $\Omega_{\text {if }}$ over a Maxwellian distribution of velocities, so that

$\Upsilon_{\text {if }}\left(T_{\mathrm{e}}\right)=\int_{0}^{\infty} \Omega_{\text {if }}\left(E_{\mathrm{f}}\right) \exp \left(-E_{\mathrm{f}} / k T_{\mathrm{e}}\right) \mathrm{d}\left(E_{\mathrm{f}} / k T_{\mathrm{e}}\right)$

where $E_{\mathrm{f}}$ is the final free electron energy after excitation and $k$ is Boltzmann's constant.

\section{Results and discussion}

The collision strengths presented in this work have been evaluated for a very fine mesh of incident impact energies, at energy intervals typically less than 0.0002 rydbergs across the energy range considered ( 0 to $10 \mathrm{Ry}$ ). This ensured that the autoionizing resonances which converge to the target state

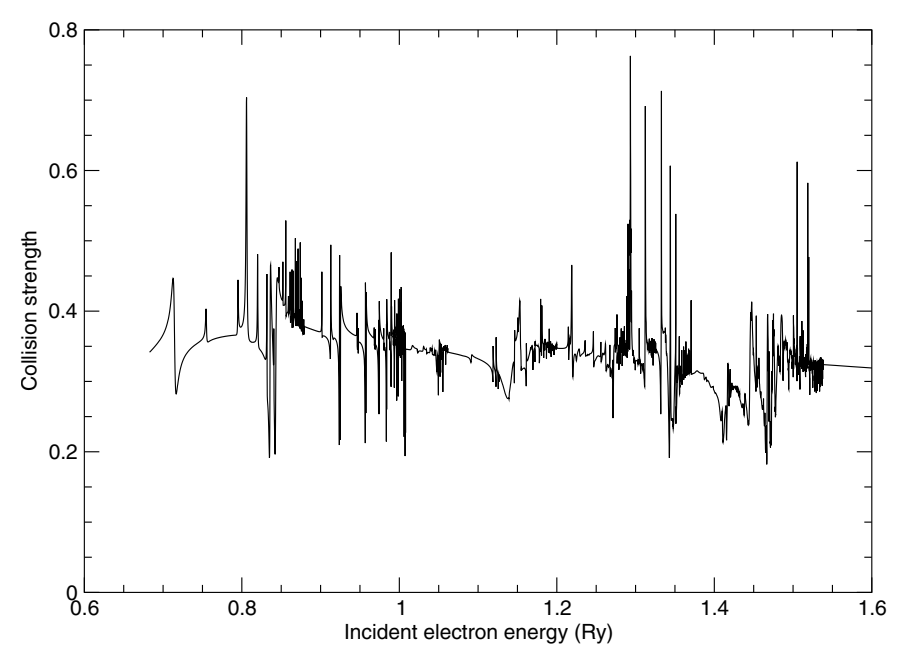

Fig. 1. Collision strength as a function of incident electron energy in rydbergs for the $2 \mathrm{~s} 2 \mathrm{p}^{2}{ }^{4} \mathrm{P}_{1 / 2}-2 \mathrm{~s} 2 \mathrm{p}^{2}{ }^{2} \mathrm{D}_{5 / 2}$ fine-structure forbidden transition.

thresholds were fully delineated. Those resonances located at energies lower than the highest threshold considered in this work $\left(2 \mathrm{~s}^{2} 4 \mathrm{f}^{2} \mathrm{~F}^{\mathrm{o}}\right)$ are physically meaningful; however at higher energies (i.e. above 1.53839 Ry) pseudo-resonances appear. These arise from the inclusion of pseudo-orbitals in the wavefunction expansion (Burke et al. 1981). At higher temperatures the high-impact energy region is much more important and so it is necessary to properly average over the pseudo-resonances to prevent distortion of the correct results in the calculation of the effective collision strengths.

In Fig. 1 we present the collision strength as a function of incident electron energy relative to the ground state in rydbergs for the $2 \mathrm{~s} 2 \mathrm{p}^{2}{ }^{4} \mathrm{P}_{1 / 2}-2 \mathrm{~s} 2 \mathrm{p}^{2}{ }^{2} \mathrm{D}_{5 / 2}$ fine-structure forbidden transition. It is evident from this figure that a large number of autoionizing resonances are present in the collision strength. The number of resonances increases as additional target states are included, since this leads to further channel-coupling effects. These resonances play a crucial role in the magnitude of Maxwellian-averaged collision strengths, normally resulting in a significant enhancement in the effective collision strength. It is therefore important that these resonances are considered carefully. The use of a suitably fine mesh of energies ensures that all resonances are fully resolved.

In Fig. 2, the effective collision strength for the $2 \mathrm{~s} 2 \mathrm{p}^{2}{ }^{4} \mathrm{P}_{1 / 2}-2 \mathrm{~s} 2 \mathrm{p}^{2}{ }^{2} \mathrm{D}_{5 / 2}$ transition from the present calculation is plotted as a function of the decimal logarithm of the electron temperature in kelvin and compared with the previous 10-state calculation of Blum \& Pradhan (1992) at a range of temperatures for which a comparison is possible. The present results are higher than those of Blum \& Pradhan (1992) by a factor of about $50 \%$ at $1000 \mathrm{~K}$, decreasing towards higher temperatures before becoming larger again. The reason for the enhancement in this calculation is the presence of additional resonances and a careful consideration of all resonances as discussed earlier.

In Fig. 3 we show the collision strength as a function of incident electron energy relative to the ground state in rydbergs for the $2 \mathrm{~s} 2 \mathrm{p}^{2}{ }^{2} \mathrm{D}_{3 / 2}-2 \mathrm{~s}^{2} 3 \mathrm{p}{ }^{2} \mathrm{P}_{3 / 2}^{\mathrm{o}}$ fine-structure allowed transition. As for the forbidden transition considered in Figs. 1 and 2, 


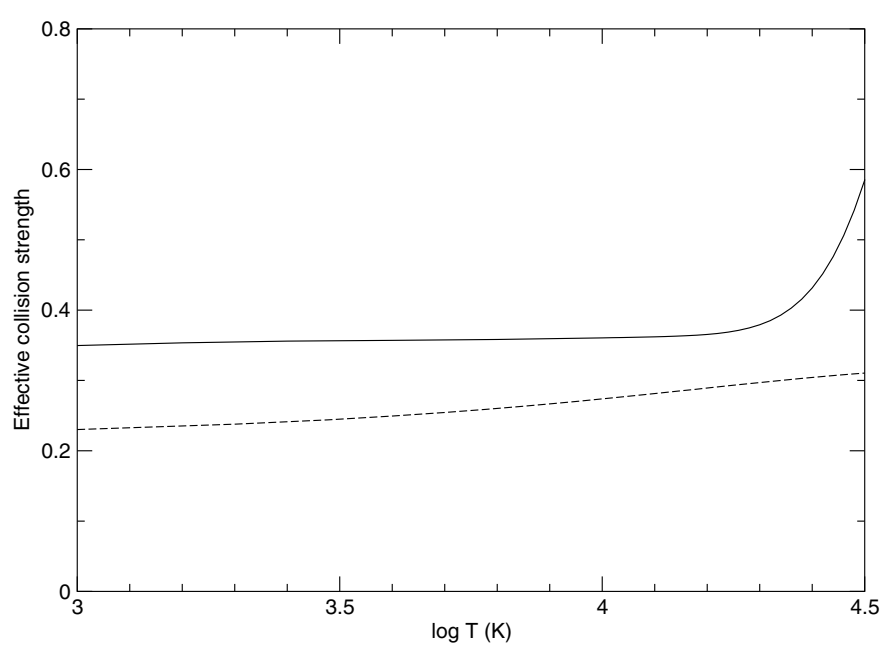

Fig. 2. Present effective collision strength as a function of $\log (T)$ where $T$ is the electron temperature in kelvin for the $2 \mathrm{~s} 2 \mathrm{p}^{2}{ }^{4} \mathrm{P}_{1 / 2}-2 \mathrm{~s} 2 \mathrm{p}^{2}{ }^{2} \mathrm{D}_{5 / 2}$ fine-structure forbidden transition (solid line) compared with the results of Blum \& Pradhan (1992) (dashed line).

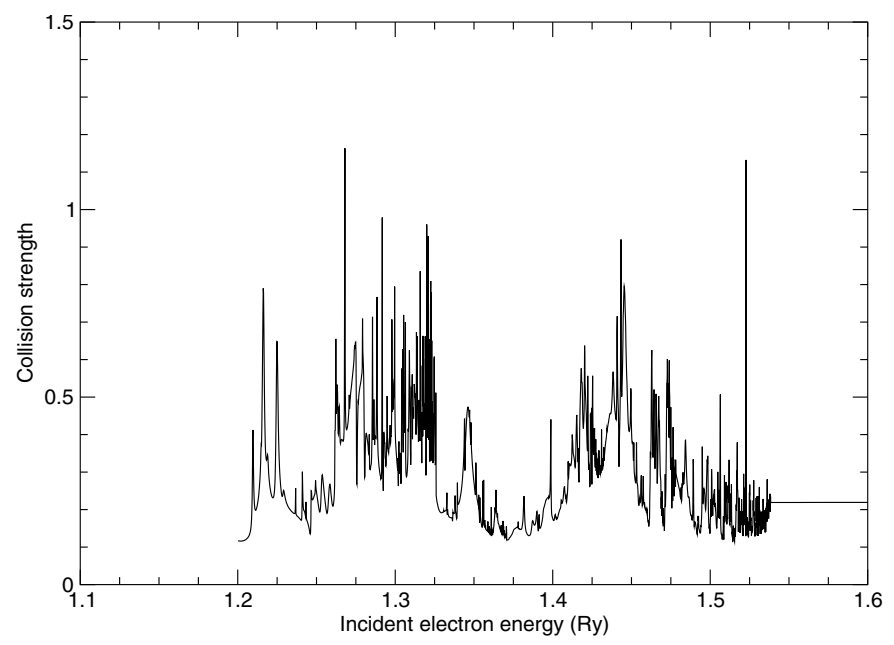

Fig. 3. Collision strength as a function of incident electron energy in rydbergs for the $2 \mathrm{~s} 2 \mathrm{p}^{2}{ }^{2} \mathrm{D}_{3 / 2}-2 \mathrm{~s}^{2} 3 \mathrm{p}^{2} \mathrm{P}_{3 / 2}^{\mathrm{o}}$ fine-structure allowed transition.

many resonant features, converging to the thresholds included in the calculation, can be observed in the collision strength spectrum. The corresponding effective collision strength from the present calculation for the $2 \mathrm{~s} 2 \mathrm{p}^{2}{ }^{2} \mathrm{D}_{3 / 2}-2 \mathrm{~s}^{2} 3 \mathrm{p}^{2} \mathrm{P}_{3 / 2}^{\mathrm{o}}$ transition is shown as the solid line in Fig. 4. As in Fig. 2, we compare our results with the calculation of Blum \& Pradhan (1992). For this allowed transition, the effective collision strengths for the two calculations are in very close agreement at lower temperatures. However at higher temperatures the present results are many times higher than the results of Blum \& Pradhan (1992).

One point, from Figs. 2 and 4, that must be commented on, is that, although there is an abundance of resonance structure in the collision strengths (Figs. 1 and 3), the corresponding effective collision strengths remain relatively flat until the high temperature extremity. This can be explained by considering the nature of Eq. (3) where we note that the exponential term

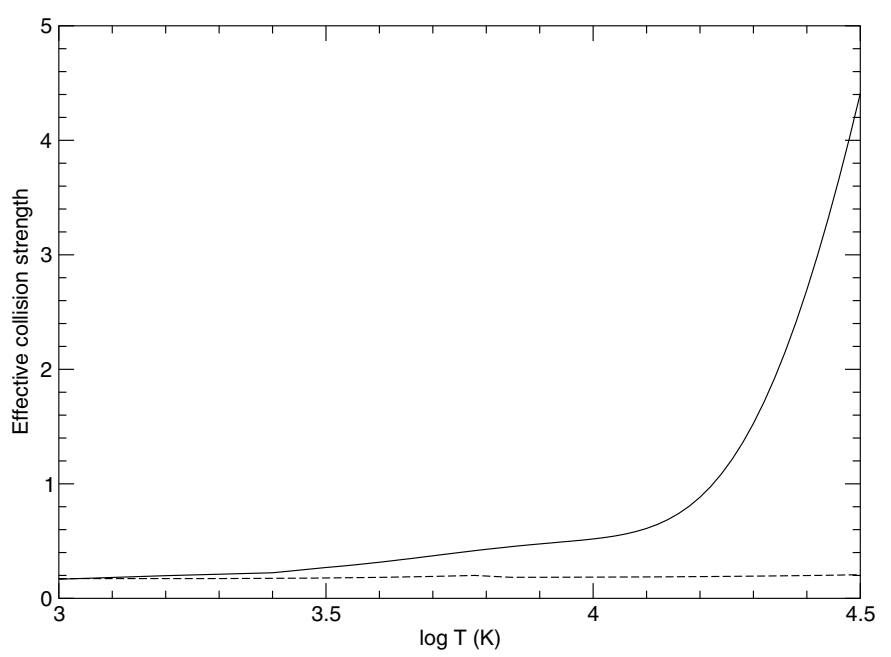

Fig. 4. The effective collision strength as a function of $\log (T)$ where $T$ is the electron temperature in kelvin for the $2 \mathrm{~s} 2 \mathrm{p}^{2}{ }^{2} \mathrm{D}_{3 / 2}-2 \mathrm{~s}^{2} 3 \mathrm{p}^{2} \mathrm{P}_{3 / 2}^{\mathrm{o}}$ fine-structure allowed transition (solid line) compared with the results of Blum \& Pradhan (1992) (dashed line).

controls how much of the collision strength is picked up when performing the integration.

This exponential term depends on the electron temperature $T_{\mathrm{e}}$ and its effect is easy to see through the following illustration. Consider an electron temperature of $\log _{10} T_{\mathrm{e}}=3$ i.e. $1000 \mathrm{~K}$. The exponential term falls off to $10 \%$ of its maximum within a energy of $0.015 \mathrm{Ry}$ above threshold. However, at $\log _{10} T_{\mathrm{e}}=4$ i.e. $10000 \mathrm{~K}$, falling off to $10 \%$ occurs after $0.15 \mathrm{Ry}$ above threshold. Thus higher temperatures allow for significant contributions to be picked up by the integral at energies further from threshold, i.e. more of the resonance structure can be picked up leading to an enhanced effective collision strength.

In Figs. 2 and 4, the apparent flatness of the effective collision strengths at low temperatures occurs because at these lower temperatures only a small energy region of the collision strength is significant and it isn't until higher temperatures that the contributions from the higher energy resonances begin to become significant. The effective collision strength in Fig. 2 is very flat until very high temperatures, which can be understood by looking at the associated collision strength in Fig. 1. Here we see that close to threshold there are very few resonances e.g looking $0.1 \mathrm{Ry}$ above threshold, we find are only two resonances in Fig. 1. Thus, it is not until the highest temperatures that a change in the effective collision strength occurs due to the contribution from resonances at energies beyond $0.1 \mathrm{Ry}$ above threshold.

The effective collision strength in Fig. 4 starts to increase more rapidly and at an earlier temperature than that in Fig. 2. This is due to more resonant structure occurring at energies closer to threshold in Fig. 3 than for the previous transition in Fig. 1. (The contrast between the two collision strengths in the energy region less than $0.1 \mathrm{Ry}$ in highly illustrative of this point.)

We also note that the values of Blum \& Pradhan (1992) shown in Figs. 2 and 4 remain flat over the entire temperature range and show no increase with temperature. This is because 


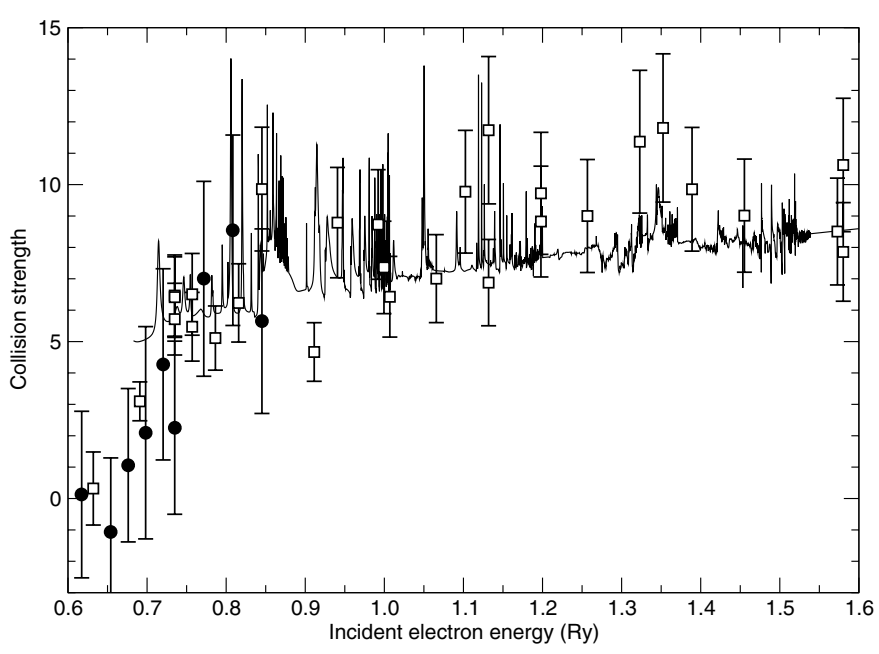

Fig. 5. Comparison of the current collision strength (solid line) with experimental data for the $2 \mathrm{~s}^{2} 2 \mathrm{p}^{2} \mathrm{P}^{\mathrm{o}}-2 \mathrm{~s} 2 \mathrm{p}^{2}{ }^{2} \mathrm{D}$ excitation: Williams et al. (1998) crossed beams (filled circles); Smith et al. (1996) merged beams (open squares).

in the current calculation the resonance structure is much more well resolved and defined.

In Fig. 5 we display the collision strength for the transition between two $L S$ levels $2 \mathrm{~s}^{2} 2 \mathrm{p}^{2} \mathrm{P}^{\mathrm{o}}-2 \mathrm{~s} 2 \mathrm{p}^{2}{ }^{2} \mathrm{D}$ and compare with experimental data. Data from two experiments have been noted: the crossed beams experiment of Williams et al. (1998) and the merged beams experiment of Smith et al. (1996). Both these experiments measure the excitation cross section, and so to make comparison with the current data we have converted the cross sections to collision strengths by applying Eq. (1).

The experiment of Williams et al. (1998) studies the near threshold excitation of the $2 \mathrm{~s}^{2} 2 \mathrm{p}^{2} \mathrm{P}^{\mathrm{o}}-2 \mathrm{~s} 2 \mathrm{p}^{2}{ }^{2} \mathrm{D}$ transition and so comparison can only be made over a small incident electron energy range. The data of Smith et al. (1996) were taken over a larger range of energies and so here we have more values with which to compare. For both sets of experimental values in the immediate threshold region $(\sim 0.68-0.75 \mathrm{Ry})$ we find that the calculated collision strength is much higher. However, once we go beyond $0.75 \mathrm{Ry}$, we find that the agreement with the experimental data becomes quite good. In fact we find that in this region there are perhaps only 2 experimental points that do not catch the calculated collision strength within the range of their error bars. This would suggest that at the very least the collision strength for this transition is known to better than $20 \%$ over this energy region. Unfortunately these appear to be the only experimental data available for this ion.

The inclusion of the $16 \mathrm{LS}$ target states leads to $30 \mathrm{~J}$ levels and a total of 435 transitions. In Table 2 (available at the CDS) we present the non-zero effective collision strengths for all transitions for a range of electron temperatures $\left[\log _{10} T_{\mathrm{e}}(\mathrm{K})=\right.$ 3.0-5.5] suitable for application in plasma and astronomical diagnostics. Table 2 contains the following information: Col. 1 lists the transitions between fine-structure levels indicated as initial-final according to the $J$-index assigned to each fine-structure level in Table 1 . For example, 2-8 denotes the transition $2 \mathrm{~s}^{2} 2 \mathrm{p}^{2} \mathrm{P}_{3 / 2}^{\mathrm{o}}-2 \mathrm{~s} 2 \mathrm{p}^{2} S_{1 / 2}$. The remaining 11 columns list the effective collision strengths for each transition at the following logarithmic electron temperatures $\log _{10} T_{\mathrm{e}}(\mathrm{K})=$ 3.00, 3.25, 3.50, 3.75, 4.00, 4.25, 4.50, 4.75, 5.00, 5.25, 5.50. A superscript indicates the power of 10 with which the number must be multiplied i.e., $a^{-n}=a \times 10^{-n}$. For some of the transitions noted in Table 2, the agreement between this calculation and the data obtained by Blum \& Pradhan (1992) is good (see for example those transitions considered in Wilson \& Bell 2002). However, as can for example be observed in Figs. 2 and 4, for other transitions significant differences are noted.

It is difficult to predict the overall accuracy of these results. However we note that we have employed sophisticated configuration-interaction wavefunctions which are in excellent agreement with experiment and have carefully considered resonant phenomena in this work. Experience from similar calculations and in particular the application of those data indicate that the effective collision strength data may be accurate to approximately $10 \%$ for transitions not involving the higherlying states. In practice the accuracy can only truly be assessed through the application of these data to plasma models or through still more sophisticated calculations.

\section{Conclusions}

In this paper we extend a previous calculation of effective collision strengths for electron impact excitation of the $\mathrm{C}$ II ion. These astrophysically important atomic data are evaluated for a large range of electron temperatures, $\left(\log _{10} T_{\mathrm{e}}(\mathrm{K})=3.0-5.5\right)$ and for all transitions among the lowest $16 \mathrm{LS}$ states of C II, corresponding to 30 fine-structure levels and 435 individual transitions. We believe that the present results are the most accurate currently available. Significant differences between the present results and the previous work of Blum \& Pradhan (1992) for some transitions are observed; for other transitions the comparison is much more favourable.

It is expected that the present effective collision strengths will have a typical accuracy of 10-20\%, particularly for those transitions between the well-represented low-lying levels. Such an accuracy is given due to the sophisticated nature of the calculation where care has been taken to included such effects as correlation, configuration-interaction and top-up of the partial waves for the allowed transitions. It should be noted, however, that uncertainties for some of the transitions between the highest lying levels of C II may be significantly larger. It is clear that either merged or crossed beams experiments are capable of producing data for comparison with theoretical values. The authors would encourage the production of such data.

Acknowledgements. The calculations were carried out using the MIRACLE supercomputer at the HiPerSPACE Computing Centre, UCL, which is funded by the UK Particle Physics and Astronomy Research Council. The work presented in this paper has also been supported by PPARC through a Rolling Grant.

\section{References}

Arad, R., Tsigutkin, K., Ralchenko, Yu. V., \& Maron, Y. 2000, Phys. Plasmas, 7, 3797 
Bell, K. L., \& Ramsbottom, C. A. 2000, Atomic Data \& Nuclear Data Tables, 76, 176

Berrington, K. A., Burke, P. G., Butler, K., et al. 1987, J. Phys. B, 20, 6379

Blum, R. D., \& Pradhan, A. K. 1992, ApJS, 80, 425

Boreiko, R. T., \& Betz, A. L. 1995, ApJ, 454, 307

Burke, P. G., \& Robb, W. D. 1975, Adv. At. Mol. Phys., 11, 143

Burke, P. G., Sukamar, C. V., \& Berrington, K. A. 1981, J. Phys. B, 14,289

Czyzak, S. J., Keyes, C. D., \& Aller, L. H. 1986, ApJS, 61, 159

Hibbert, A. 1975, Comput. Phys. Comm., 9, 141

Hyung, S., Aller, L. H., Feibelman, W. A., \& Lee, S.-J. 2001, ApJ, 563,889
Johnson, H. R., Ensman, L. M., Alexander, D. R., et al. 1995, ApJ, 443,281

Ramsbottom, C. A., Bell, K. L., \& Keenan, F. P. 2001, Atomic Data \& Nuclear Data Tables, 77, 57

Saraph, H. E. 1978, Comput. Phys. Comm., 15, 247

Smith, S. J., Zuo, M., Chutjian, A., Tayal, S. S., \& Williams, I. D. 1996, ApJ, 463, 808

Trapero, J., Welty, D. E., Hobbs, L. M., et al. 1996, ApJ, 468, 290

Vassiliadis, E., Dopita, M. A., Bohlin, R. C., et al. 1998, ApJS, 114, 237

Williams, I. D., Greenwood, J. B., Srigengan, B., O’Neill, R. W., \& Hughes, I. G. 1998, Meas. Sci. Technol., 9, 930

Wilson, N. J., \& Bell, K. L. 2002, MNRAS, 337, 1027 